Research Article

\title{
Discussion on the Theoretical Basis for Cross-Over Method Applied to Downhole Wave Velocity Test
}

\author{
Qing Dong $\mathbb{D}^{D}$, Zheng-hua Zhou $\mathbb{D}^{\mathbb{D}}$, Su Jie $\mathbb{i}$, Bing Hao, and Yuan-dong Li \\ College of Transportation Science \& Engineering, Nanjing Tech University, Nanjing, China \\ Correspondence should be addressed to Zheng-hua Zhou; bjsmoc@163.com
}

Received 30 April 2021; Accepted 3 June 2021; Published 15 June 2021

Academic Editor: Kaiming Bi

Copyright ( 2021 Qing Dong et al. This is an open access article distributed under the Creative Commons Attribution License, which permits unrestricted use, distribution, and reproduction in any medium, provided the original work is properly cited.

\begin{abstract}
At engineering practice, the theoretical basis for the cross-over method, used to obtain shear wave arrival time in the downhole method of the wave velocity test by surface forward and backward strike, is that the polarity of P-wave keeps the same, while the polarity of S-wave transforms when the direction of strike inverted. However, the characteristics of signals recorded in tests are often found to conflict with this theoretical basis for the cross-over method, namely, the polarity of the P-wave also transforms under the action of surface forward and backward strike. Therefore, 3D finite element numerical simulations were conducted to study the validity of the theoretical basis for the cross-over method. The results show that both shear and compression waves are observed to be in $180^{\circ}$ phase difference between horizontal signal traces, consistent with the direction of excitation generated by reversed impulse. Furthermore, numerical simulation results prove to be reliable by the analytic solution; it shows that the theoretical basis for the cross-over method applied to the downhole wave velocity test is improper. In meanwhile, numerical simulations reveal the factors (inclining excitation, geophone deflection, inclination, and background noise) that may cause the polarity of the P-wave not to reverse under surface forward and backward strike. Then, as to reduce the influence factors, we propose a method for the downhole wave velocity test under surface strike, the time difference of arrival is based between source peak and response peak, and numerical simulation results show that the S-wave velocity by this method is close to the theoretical S-wave velocity of soil.
\end{abstract}

\section{Introduction}

S-wave velocity $\left(V_{S}\right)$ of site soil is a key dynamic parameter in seismic engineering, mainly reflecting site dynamic property determination, site seismic response analysis, and site classification [1-5]. Many in situ seismic tests such as noninvasive methods (single or multichannel spectral analysis of surface waves) and invasive methods (crosshole or downhole method and PS logging) are now generally used for obtaining $V_{S}$ of soil layers under a small strain level [3,6-10]. Generally, it is deemed that, by simple arithmetic, invasive methods can obtain more reliable $V_{S}$ in a thick overlayer site compared to the noninvasive methods which calculate $V_{S}$ using an inversion process and are of multiple solutions. Among invasive methods, the downhole method under surface forward and backward strike is widely used for its several advantages such as fewer boreholes than that of crosshole method and convenient operation.

In the downhole method, after the surface excitation, body waves propagate in the soil layers and are recorded by receivers inside the borehole. Based on the signals, the travel time of the propagation of body waves in soil layers can be calculated, which will be finally used for the $V_{S}$ profile of the site. Therefore, it precisely determines the travel time of Swave. Generally, estimating the travel time of the S-wave from a single waveform is difficult since the arrival time of the S-wave is not always clear. To overcome the drawback of the single waveform, the cross-over method is frequently used in practice for the identification of S-wave arrival time through a forward and backward surface excitation [11, 12]. The theoretical basis of the cross-over method is that the polarity of the P-wave keeps the same, while the polarity of the S-wave changes when the direction of striking is 
inverted. However, in in situ tests, $V_{S}$ obtained directly according to the cross-over method is of significant error. On one hand, the arrival time of the S-wave in two sets of signals recorded by the forward and backward strike at the same depth is inconspicuous owing to the environmental noise or the weak energy of strike $[13,14]$. On the other hand, the onset $\mathrm{P}$-wave component with reversed polarity and small amplitude appears in some signals with a clear $S$-wave component $[6,15]$, thus giving rise to the misjudgment of the real cross-over point of S-waveform. All these issues would cause significant errors between evaluation S-wave velocity and the actual one. For the environmental noise, it can be eliminated by the enhancement of the signal-to-noise ratio or the selection of less interfered testing moment. However, the reversed part of the signal before the arrival of the S-wave is often ignored due to its small amplitude, and its characteristics were rarely investigated. Therefore, it is of great engineering significance for reliable determination of onshore or offshore engineering site property that the characteristics of signals recorded in the downhole method are deeply studied. In the meantime, this study provides a theoretical basis for the judgment of S-wave onset and better applies the cross-over method to the S-wave velocity test of onshore or offshore engineering sites.

Herein, a three-dimensional (3D) lumped-mass finite element model is established to simulate the downhole method of the $V_{S}$ test. By the time-domain dynamic analysis method with explicit step-by-step integration, the vibration responses under surface excitation are obtained and used for the analysis of the characteristics of signals recorded at different observation depths and the rationality of the crossover method. In addition, the reliability of numerical simulation results and the validity of the theoretical basis of the cross-over method applied to the downhole method are verified by the analytic solution. Finally, based on the numerical simulation results, a method for calculating S-wave travel time is proposed and can obtain more accurate $V_{S}$ with less influence by in situ test conditions. This effort is conducive to understand the theoretical basis of the crossover method well and to determinate the travel time of the S-wave precisely.

\section{Discussion on the Theoretical Basis for Cross- Over Method}

In the downhole method, a probe constructed with three geophone elements in an $X-Y-Z$ orthogonal configuration sealed in a cylindrical package is placed inside the borehole. By horizontally striking the end of both sides of the plank (see Figure 1(a)), a set of excitations of opposite directions are generated. Then, the cross-over method, with the theoretical basis that only the polarity of the S-wave is reversed, is used for the interpretation of the signals received by the horizontal geophone to obtain the S-wave travel time.

$V_{S}$ obtained in site tests by the cross-over method is not always satisfactory and is easily influenced by "picking time." Figure 1(b) gives partial testing results of the downhole method in a shallow overburden site in Miyun District, Beijing. By the cross-over method, the green dot in
Figure 1(b) could be suggested as the first arrival of the S-wave under the consideration of the initial polarized part. However, the blue dot would be selected as the arrival of the S-wave eventually, combined with the characteristic of S-wave and $\mathrm{P}$-wave amplitude. In two cases, the shear wave velocity calculated by the blue dot is about $489.1 \mathrm{~m} / \mathrm{s}$, which is closer to the empirical $V_{S}$ value of cobble than that calculated by the green dot. It shows a situation that if the blue dot was picked as the arrival of the $\mathrm{S}$-wave, the reversed signal before the blue dot should be P-wave, which conflicts with the theoretical basis of the cross-over method. From this, what are the characteristics of signals received in the downhole method and whether is the theoretical basis of the cross-over method rational? To a better explanation of the abovementioned questions, a 3D lumped-mass finite element model was established to simulate the downhole method of the shear wave velocity test and resolved by the time-domain dynamic analysis method with explicit step-by-step integration.

\section{Finite Element Numerical Simulation of the Downhole Method}

To simulate the signals generated by the surface forward and backward excitation in a downhole method, a 3D analysis model was established. A computational region with a size of $16 \mathrm{~m} \times 16 \mathrm{~m} \times 25 \mathrm{~m}$ was cut out from a uniform isotropic semi-infinite space Earth medium by introducing an artificial boundary condition. The upper boundary is free; the bottom boundary and the lateral boundaries are artificial boundaries. According to Campanella and Stewart [16] and Ishihara [17], soil shear strains during the downhole method of the wave velocity test are at a small strain level, thus the soil medium is linear elasticity, and the medium has parameters of $V_{S}=200 \mathrm{~m} / \mathrm{s}$, density $\rho=1900 \mathrm{~kg} / \mathrm{m}^{3}$, and Poisson's ratio $\mu=0.45$. The hammer strike was simulated by a horizontal $X$-direction impulse excitation on the center of the model surface, and the cut-off frequency of the excitation is about $80 \mathrm{~Hz}$ (see Figure 1(c)). According to the dynamic finite element computational precision, the analysis model was discretized by a 3D eight-node hexahedral solid finite element of size $0.2 \mathrm{~m} \times 0.2 \mathrm{~m} \times 0.2 \mathrm{~m}$. The time step was determined as $0.0002 \mathrm{~s}$ based on the stability condition of the time-domain explicit dynamic finite element method [18]. The Multitransmitting Formula (MTF) [18-20] was utilized in the artificial boundaries of the model to simulate the effect of radiation damping in elastic half-space, for ensuring the nonreflection of the scattered waves when passing through the artificial boundaries from the computational region. Parameters such as unit size and time step set in the numerical calculation of the model are selected according to the stability criterion of numerical integration [18], in which the unit size meets the following equation:

$$
\Delta x \leq\left(\frac{1}{6} \sim \frac{1}{10}\right) \frac{V_{S}}{f},
$$

where $\Delta X$ is the size of the unit, $V_{s}$ is the S-wave velocity of the model, and $f$ is the maximum frequency of input excitation with engineering significance. 

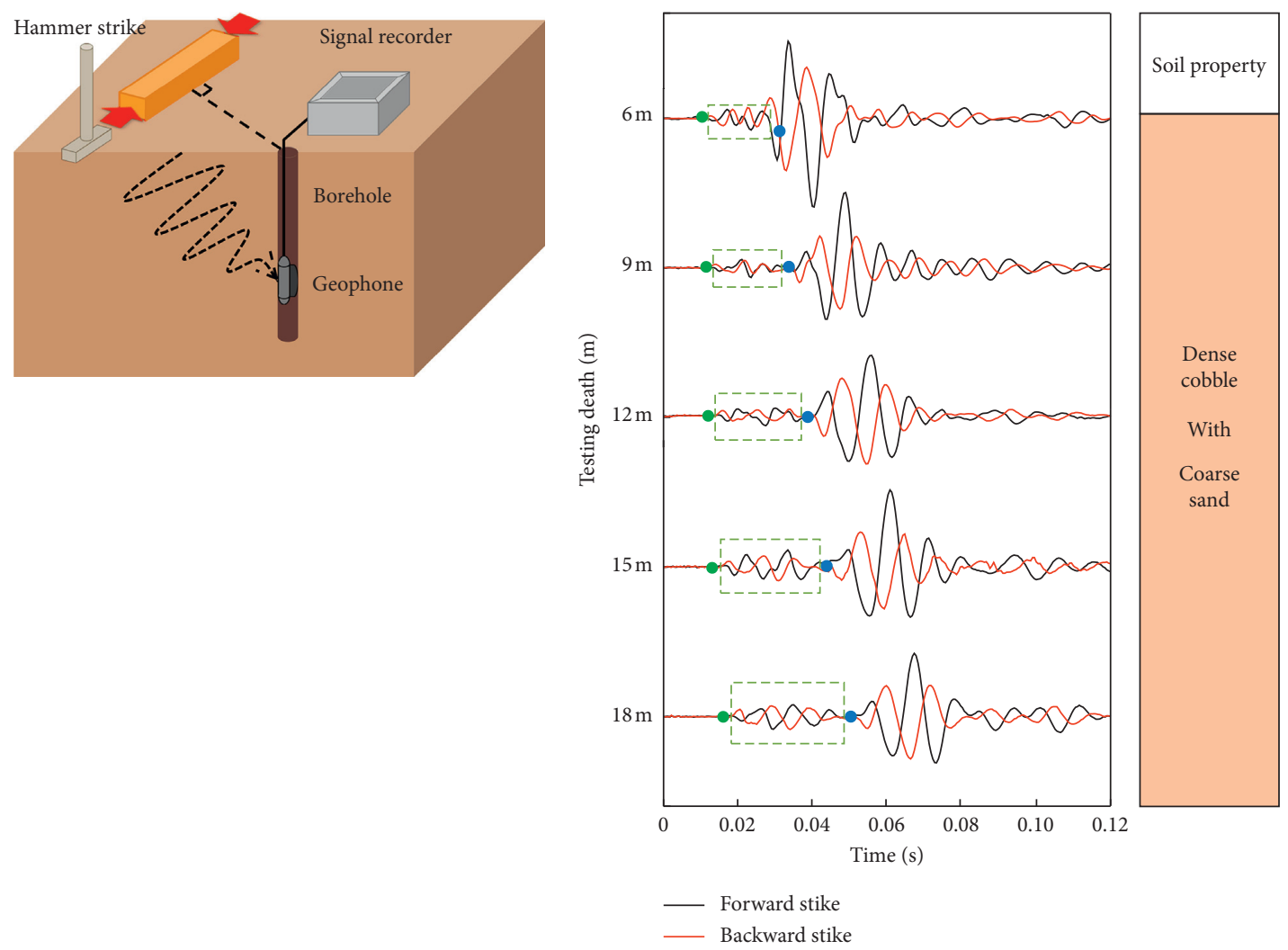

(a)

(b)

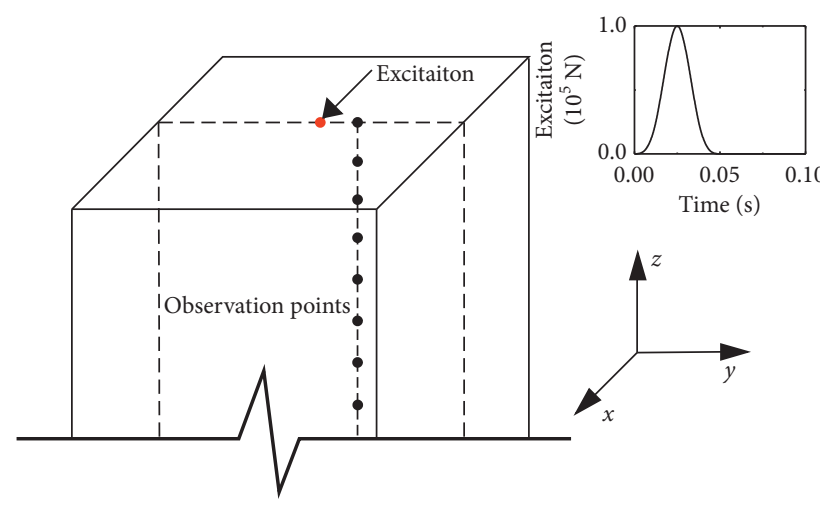

(c)

Figure 1: Downhole method. (a) Schematic of the downhole velocity test method with the surface plank source. (b) Signal traces recorded at different testing depth by the downhole method at a site in Beijing. (c) $3 \mathrm{D}$ numerical analysis model of size $16 \mathrm{~m} \times 16 \mathrm{~m} \times 25 \mathrm{~m}$.

The time step $\Delta t$ should also meet the following stability conditions:

$$
\Delta t \leq \min \left(\frac{\Delta x}{V}\right),
$$

where $V$ is the velocity of the $\mathrm{P}$-wave in the $3 \mathrm{D}$ model.

Figure 2 gives the signal traces at observation points with different depths in horizontal $X$ direction under the forward and backward excitation. The polarities of all signals are reversed when the opposite excitations are applied, as shown in Figures 2(a)-2(c)). The time of the first cross-over point judged by the cross-over method in the signal traces, marked as $T_{A}$, is regarded as the arrival time of the $\mathrm{S}$-wave, but this is not the case.

It is known from the theoretical analysis that, in a uniform isotropic infinite medium, there is no pure $S$-wave because of the inherent coupling between the $\mathrm{P}$-wave and S-wave generated by the point source $[21,22]$. According to the wave velocity of the medium in the model, the accurate arrival times of the $\mathrm{P}$-wave and $S$-wave at observation points with different depths can be calculated and were marked as $T_{P}$ and $T_{S}$ in signal traces, respectively (as shown in Figure 2). Compared with $T_{P}$ and $T_{S}$, it is found that $T_{A}$ is between $T_{P}$ and $T_{S}$, and $V_{S}$ would be overestimated by $T_{A}$. Besides, it is seen from the 
front part of signal traces amplified at $8 \mathrm{~m}, 16 \mathrm{~m}$, and $24 \mathrm{~m}$ observation points, as shown in Figures $2(\mathrm{~d})-2(\mathrm{f})$, that the initial P-wave also shows the reversed polarity under the forward and backward excitation. Therefore, the judgment of the arrival time of the S-wave using the cross-over method is inaccurate.

As observed in Figure 2, when the observation points are at shallow depths, S-wave onset is disturbed by the $\mathrm{P}$-wave which arrived earlier and is hard to be identified. Amplifying the waveform also does not help much (as shown in Figure 2(d)); the identification of $T_{P}$ can be still mistaken as $T_{S}$ easily. With the observation point's depth increasing, the P-wave gradually separates with the $\mathrm{S}$-wave since $\mathrm{P}$-wave velocity is larger than $V_{S}$ (see Figure 2(f)). Although the P-wave decays sharply with the increase of depth, its energy still affects S-wave onset, thus the judgment of $T_{S}$ is still difficult. Under this situation, only the approximate S-wave onset would be identified even enlarging the signals, and it is of different value with different scales. In addition, it is difficult to identify P-wave onset because of the relatively small amplitude of the P-wave compared with the

$$
\left\{\begin{array}{l}
u=0, \\
u=\frac{F}{4 \pi \mu Z} \frac{\tau\left(\tau^{2}-(1 / 3)\right)\left(\tau^{2}+(2 / 3)\right)^{1 / 2}}{\left(2 \tau^{2}+(1 / 3)\right)^{2}-4 \tau\left(\tau^{2}-(1 / 3)\right)\left(\tau^{2}+(2 / 3)\right)^{1 / 2}}, \\
u=\frac{F}{4 \pi \mu Z}\left\{\left(\tau^{2}+1\right)-\frac{2 \tau\left(\tau^{2}-(1 / 3)\right)\left(\tau^{2}+(2 / 3)\right)^{1 / 2}}{\left(2 \tau^{2}+(1 / 3)\right)^{2}-4 \tau\left(\tau^{2}-(1 / 3)\right)\left(\tau^{2}+(2 / 3)\right)^{1 / 2}}+\frac{2 \tau^{2}\left(\tau^{2}-1\right)\left[2 \tau\left(\tau^{2}-(2 / 3)\right)^{1 / 2}-\left(2 \tau^{2}-1\right)\right]}{\left(2 \tau^{2}-1\right)^{2}-4 \tau\left(\tau^{2}-1\right)\left(\tau^{2}-(2 / 3)\right)^{1 / 2}}, \quad 1 / \sqrt{3}<\tau<1,\right. \\
1<\tau,
\end{array}\right.
$$

where

$$
\tau=\frac{V_{s} t}{Z},
$$

and $F$ is the Heaviside unit function, $Z$ is the distance from the load point, $\mu$ is the shear moduli, and $V_{S}$ is the shear wave velocity.

To obtain the numerical solution of equation (3), an approximate Heaviside unit function corresponding to the Heaviside unit function in equation (3) is introduced, as shown in the following equation:

$$
F(\bar{t})= \begin{cases}16 \bar{t}^{3}, & 0.0 \leq \bar{t}<0.25 \\ 1-48 \bar{t}(\bar{t}-0.5)^{2}, & 0.25 \leq \bar{t}<0.5, \\ 1, & \bar{t} \geq 0.5,\end{cases}
$$

where $\bar{t}$ is dimensionless time, $\bar{t}=0.5 t / t_{0}, t$ is the time factor, and $t_{0}$ is the rise time of the approximate unit Heaviside function. When $t_{0}=0.05 \mathrm{~s}$, the approximate Heaviside unit function is shown in Figure 3(a). It is not difficult to prove
S-wave, which gives rise to wrong engineering cognitions that the P-wave has no polarized characteristics.

The theoretical results illustrate that the polarity of the $\mathrm{P}$-wave cannot keep the same when the $\mathrm{S}$-wave reversed the polarity under the backward excitation. However, it can be observed in in situ tests that the P-wave has the same polarity under the forward and backward excitation, which probably is because of the influence of testing status and environmental interference.

\section{Analytical Validation of Theoretical Basis for Cross-Over Method}

Based on exact and closed-form solutions of a homogeneous isotropic elastic half-space due to the application of a varying with time as the Heaviside unit function by Chao [23] and with the aid of Laplace and Hankel transforms, the displacements in the direction consistent with load direction directly below the applied tangential point force acted on the surface, corresponding Poisson's ratio $=1 / 4$, could be represented as equation (3) by the semi-inverse method: concentrated force tangential to the plane boundary and from (5) that $F(\bar{t})$ is continuous and differentiable, and its first-order derivatives are continuous in the region of $\bar{t} \geq 0$.

By changing the polarity of $F(\bar{t})$, two sets of displacements corresponding to different $Z$ of $2 \mathrm{~m}$ and $8 \mathrm{~m}$ distances directly below the load point were calculated by the equation in which $\mu=68 \mathrm{MPa}$ and $V_{S}=200 \mathrm{~m} / \mathrm{s}$, as shown in Figure 3(b). The arrival time $t$ is nondimensionalized by $V_{S} t / Z$. In this way, the value 1.0 of the abscissa corresponds to the arrival of the S-wave, while the value of 0.577 corresponds to the arrival of the $\mathrm{P}$-wave, as shown in Figure 3(b). It can be observed that the first cross-over point of two traces is the arrival of the $\mathrm{P}$-wave, and the $\mathrm{P}$-wave is exclusively reversed when the polarity of $F(\bar{t})$ is opposite.

Though the analytical solution cannot simulate the complex geometry of the downhole method, it is helpful to understand the characteristics of P-wave initial motion, which is consistent with the simulated result above. Therefore, when the direction of the impulse is reversed, both $\mathrm{P}$ - and S-waves should reverse the polarity. Thus, it can be reached that the theoretical basis for the cross-over method applied to the downhole method is improper. 


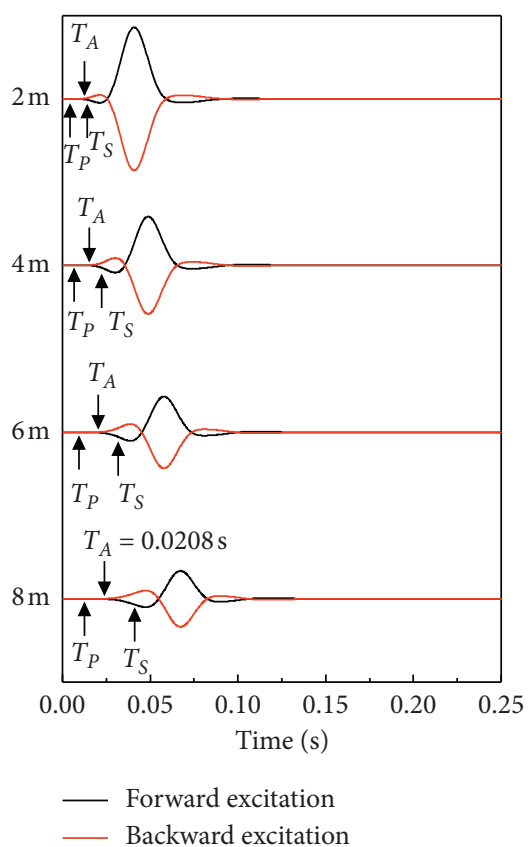

(a)

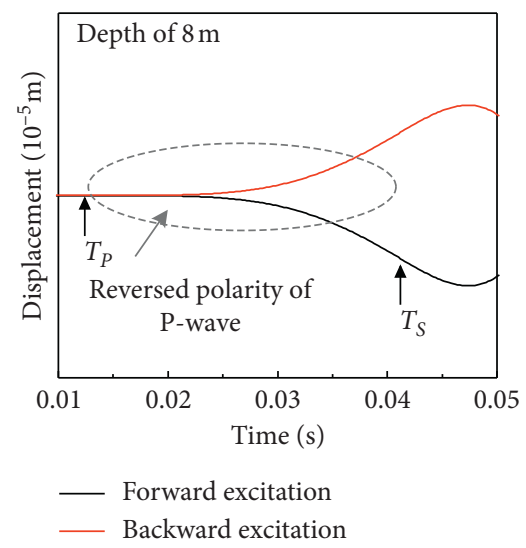

(d)

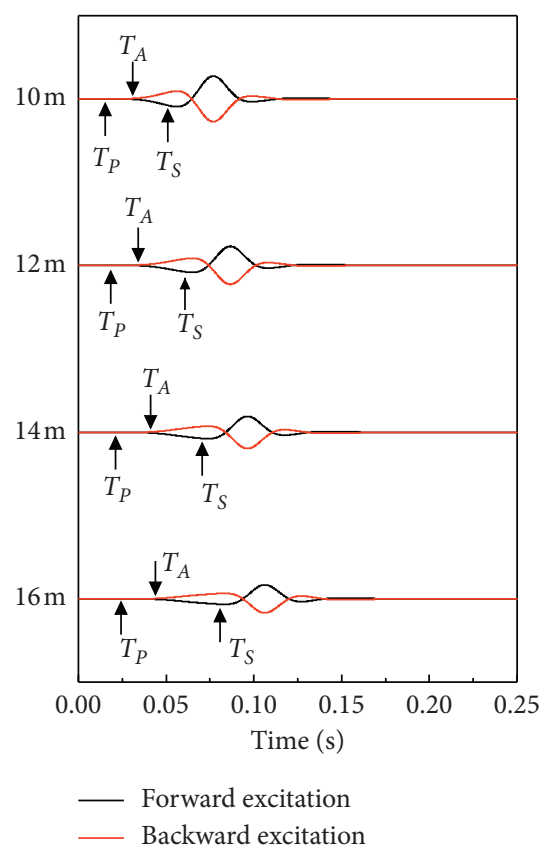

(b)

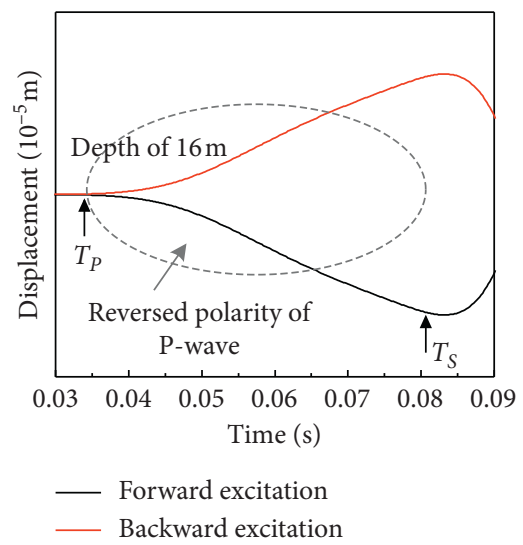

(e)

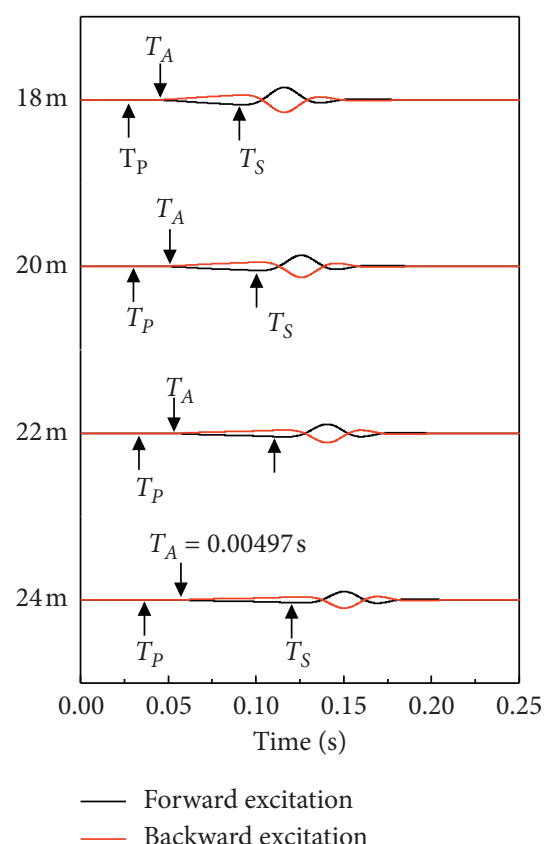

(c)

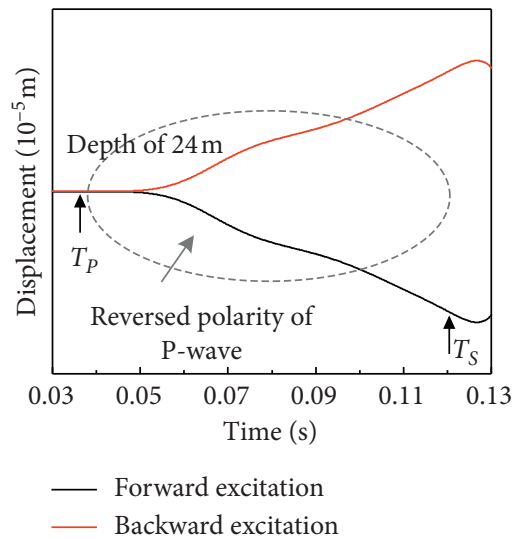

(f)

Figure 2: Signal traces at different depth observation points in the horizontal $X$ direction under the forward and backward excitation; (a)-(c) the original signal traces at different selected depth; (d)-(f) amplified waveform of partially signals at $8 \mathrm{~m}, 16 \mathrm{~m}$, and $24 \mathrm{~m}$ observation points.

\section{Calculation Methods of Shear Wave Velocity in Downhole Method}

The above findings show that the polarity of the P-wave is reversed when the direction of excitation is inverted; therefore, the arrival time of the S-wave cannot be accurately and efficiently identified according to the cross-over method. Besides the cross-over method, two other methods can be used for S-wave travel time calculation in the downhole method, i.e., peak-to-peak method (PPM) and cross-correlation method (CCM) $[16,24-26]$. Both methods can obtain reliable $V_{S}$ when the waveform of the signal is of obvious S-wave peak. However, the correlation between the signals of adjacent observation points is poor when only one receiver receives the signal at each strike. In addition, due to the influence of inclination of excitation, deflection, inclination of the geophone on the peak position, and amplitude of the signal, the accuracy of the travel time calculated by PPM is affected.

Influenced by the inclination of excitation, deflection, and inclination of the geophone, the S-wave will be detected in three geophone elements which are in an $X-Y-Z$ orthogonal configuration. Therefore, a new S-wave travel time calculation method is proposed. Firstly, a root mean square signal of two horizontal components and a vertical component in the timedomain is acquired by equation (6). Then, referring to the common peak-peak method, the peak of the waveform of the composite signals is selected which is quite representative of S-wave motion. By calculation of the time shift between the peak of trigger signals and the peak of the composite signals or the peaks of the composite signals of adjacent observation points, the S-wave travel time can be acquired, which is shown in Figure 4: 


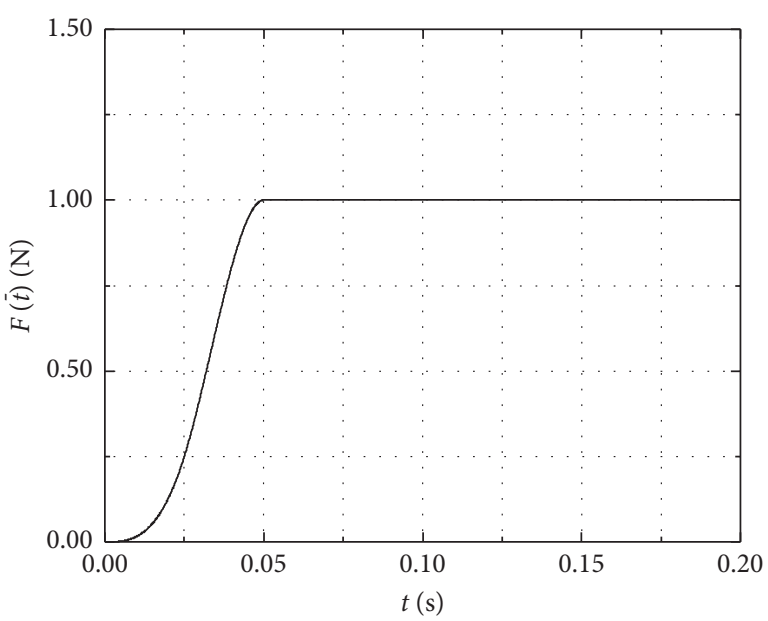

(a)

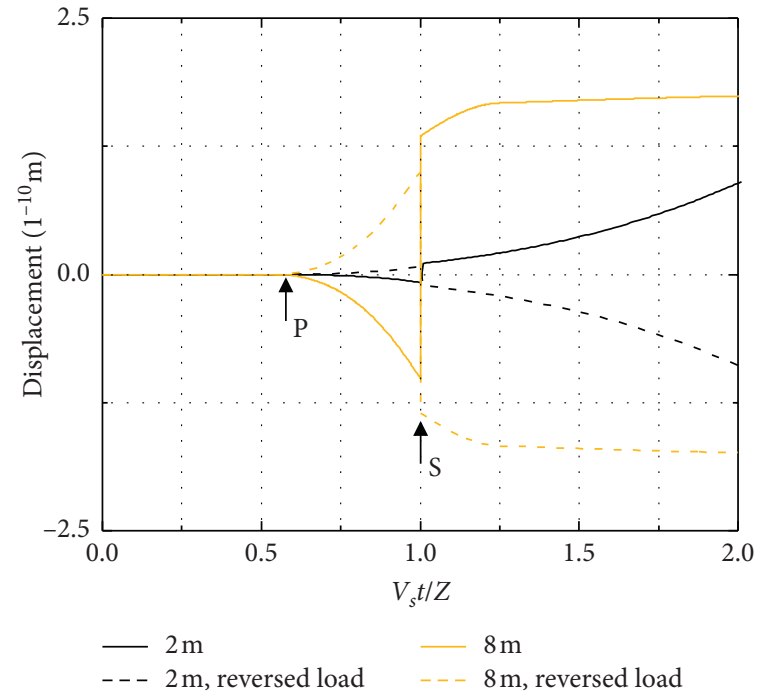

(b)

Figure 3: Analytical method result. (a) Approximate Heaviside unit function used for the analytical method. (b) Displacements of $2 \mathrm{~m}$ and $8 \mathrm{~m}$ in an elastic half-space directly below the surface tangential point source. Poisson's ratio is 0.25 .

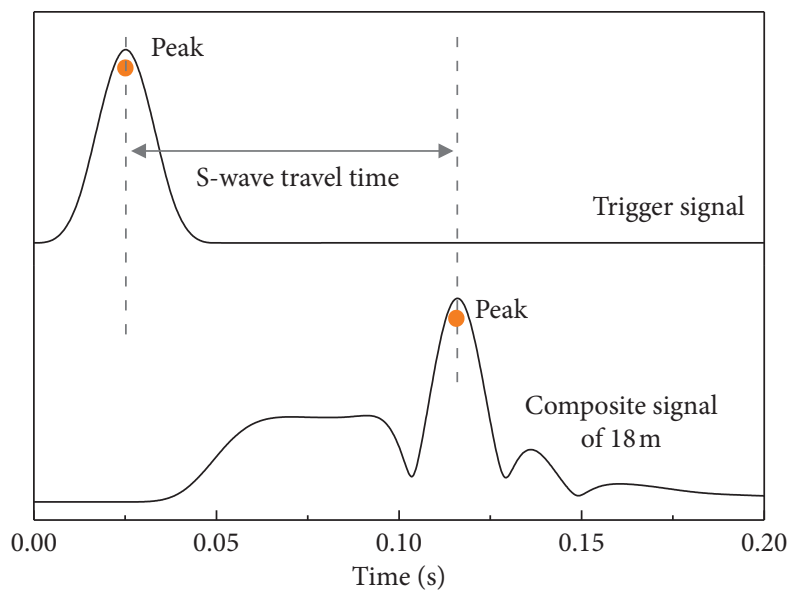

FIgURE 4: Schematic of NPPM for S-wave travel time calculation.

$$
\operatorname{Signal}_{\mathrm{MIX} 3}=\frac{\sqrt{\left(\operatorname{Signal}_{\mathrm{H}_{1}}^{2}+\operatorname{Signal}_{\mathrm{H}_{2}}^{2}+\operatorname{Signal}_{\mathrm{V}}^{2}\right)}}{3} .
$$

The travel times of S-wave propagating between the source and observation point and between adjacent $1 \mathrm{~m}$ interval observation points were calculated through PPM, CCM, and NPPM, respectively. According to the velocity equation, S-wave velocity was calculated. Figure 5 displays relative errors between $V_{S}$ of the medium in the model and calculated $V_{S}$ under the conditions of inclined excitation, geophone deflection, and inclination. For the $V_{S}$ error calculation of PPM and CCM, signals in two horizontal directions (signed as $\mathrm{NH}_{1}$ and $\mathrm{NH}_{2}$ ) were used.

As observed, under the combination of a variety of conditions, the relative error of $V_{S}$ by NPPM is less than $5 \%$ when the depth is more than $3 \mathrm{~m}$, which is even less than $2.5 \%$ by using the trigger signal. As for the result through the PPM and CCRM, the relative error of the S-wave within $9 \mathrm{~m}$ in Figure 5 gradually increases with the depth decreasing and up to $10 \%$ in the depth of $5 \mathrm{~m}$. Moreover, it can be seen from Figures 5(a) and 5(c) that the calculation accuracies of PPM and CCRM are significantly affected by the deflection of the geophone and the selection of horizontal signals. When the inclination angle of the excitation change from $20^{\circ}$ to $45^{\circ}$ (shown in Figures 5(a)-5(f)), the travel time calculated by PPM and CCRM is significantly affected and the corresponding relative error of $V_{S}$ increases. The results indicate that the proposed new peak-to-peak method (NPPM) can reduce the effects of inclined excitation, geophone deflection, and inclination on the calculation of shear wave travel time, and the determined $V_{S}$ is closed to the real one. 


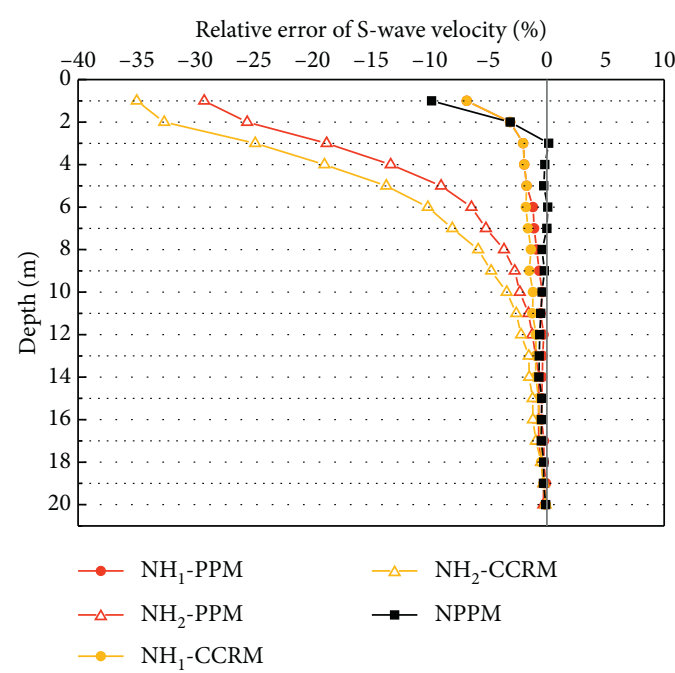

(a)

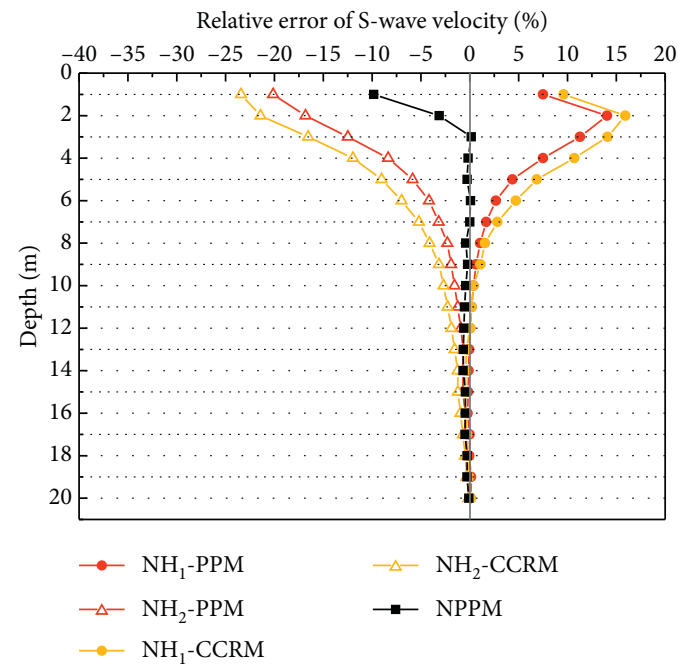

(c)

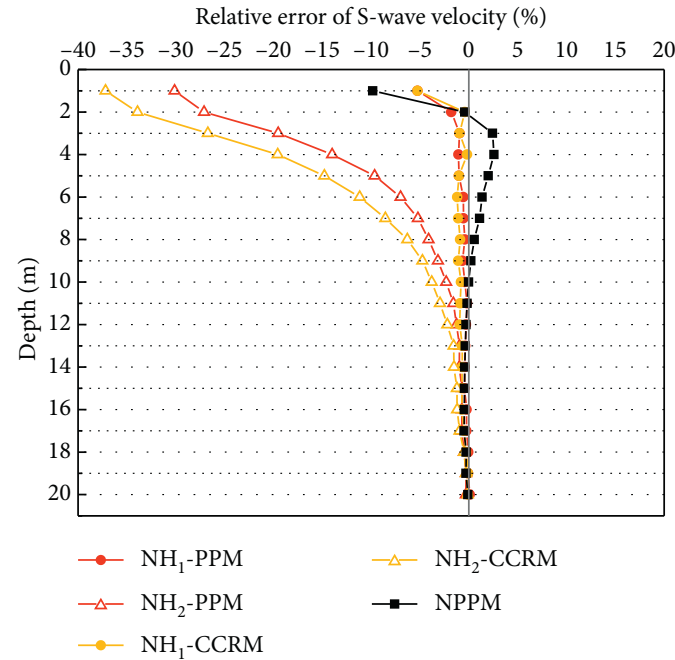

(e)

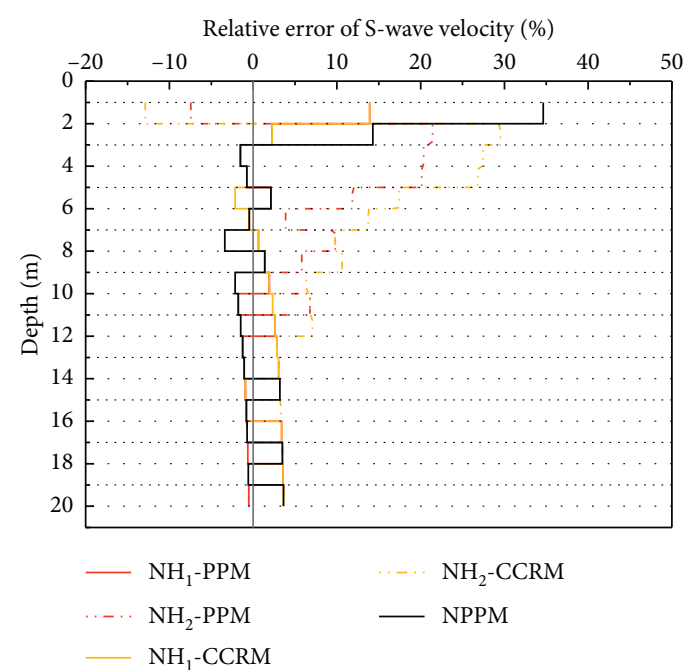

(b)

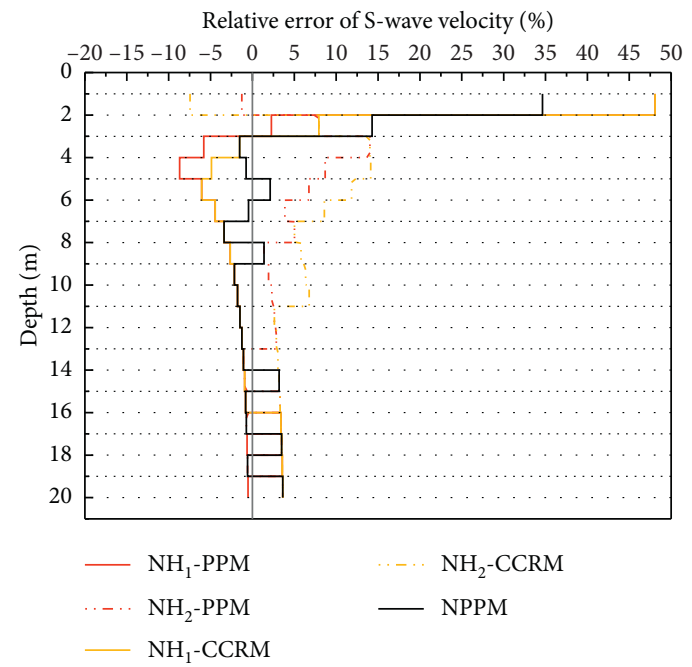

(d)

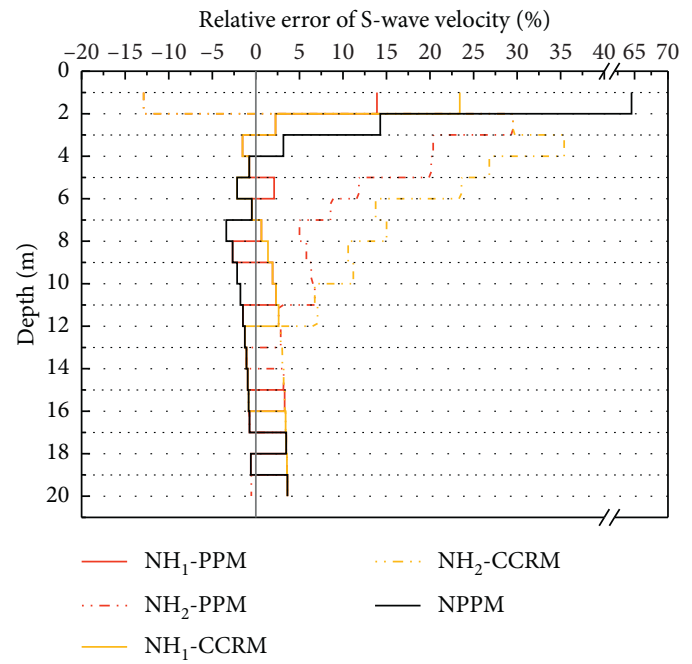

(f)

FIGURE 5: Relative error of $V_{S}$ of PPM, CCM, and NPPM at different testing depths: (a) excitation inclination $20^{\circ}$, geophone deflection $20^{\circ}$, and geophone inclination $10^{\circ}$, using the trigger signals; (b) excitation inclination $20^{\circ}$, geophone deflection $20^{\circ}$, and geophone inclination $10^{\circ}$, based on the adjacent received signals; (c) excitation inclination $20^{\circ}$, geophone deflection $50^{\circ}$, and geophone inclination $10^{\circ}$, using the trigger signals; (d) excitation inclination $20^{\circ}$, geophone deflection $50^{\circ}$, and geophone inclination $10^{\circ}$, based on the adjacent received signals; (e) excitation inclination $45^{\circ}$, geophone deflection $20^{\circ}$, and geophone inclination $10^{\circ}$, using the trigger signals; (f) excitation inclination $45^{\circ}$, geophone deflection $20^{\circ}$, and geophone inclination $10^{\circ}$, based on the adjacent received signals. 


\section{Conclusions}

To study the reliability of the cross-over method which is applied to the downhole method of off-shore and on-shore site wave velocity test, we adopted a 3D time-domain explicit dynamic finite element model combined with MTF to investigate the P-/S- wave velocity of the site based on the cross-over method presented in this paper. The conclusions and findings are as follows:

(1) The results in this paper show that the theoretical basis, the polarity of $\mathrm{P}$-wave keeps the same, while the S-wave polarity inverses with surface forward and backward strike, is invalid

(2) The numerical simulation of this paper shows that the conditions for the theoretical foundation of the cross-over method are caused by the factors of the inclining excitation, geophone deflection, inclination, and background noise

(3) Based on our numerical simulation and analytical solution, the time difference of arrival based between source peak and response peak and the calculation method of S-wave velocity are proposed in this paper

\section{Data Availability}

The data used to support the findings of this study are available from the corresponding author upon request.

\section{Conflicts of Interest}

The authors declare that there are no conflicts of interest regarding the publication of this paper.

\section{Authors' Contributions}

Dong Qing, Zhou Zhenghua, and Jie Su contributed to the concept and design of the study. Dong Qing contributed to the analysis and interpretation of data. Dong Qing wrote the draft of the manuscript. Zhou Zhenghua did the critical revision of the manuscript for important intellectual content. Hao Bing and Li Yuan-Dong performed statistical analysis. Zhou Zhenghua obtained funding. Zhou Zhenghua supervised the study.

\section{Acknowledgments}

This work was supported by the National Natural Science Foundation of China (U2039208 and U1839202) and Postgraduate Research and Practice Innovation Program of Jiangsu Province.

\section{References}

[1] A. Kayabasi and C. Gokceoglu, "Liquefaction potential assessment of a region using different techniques (Tepebasi, Eskişehir, Turkey)," Engineering Geology, vol. 246, pp. 139-161, 2018.

[2] J. A. Schneider, P. W. Mayne, and G. J. Rix, "Geotechnical site characterization in the greater memphis area using cone penetration tests," Engineering Geology, vol. 62, no. 1-3, pp. 169-184, 2001.

[3] J. A. Hunter, S. E. Pullan, R. A. Burns et al., "Downhole seismic logging for high-resolution reflection surveying in unconsolidated overburden," Geophysics, vol. 63, no. 4, pp. 1371-1384, 1998.

[4] J. A. Hunter, B. Benjumea, J. B. Harris et al., "Surface and downhole shear wave seismic methods for thick soil site investigations," Soil Dynamics and Earthquake Engineering, vol. 22, no. 9-12, pp. 931-941, 2002.

[5] R. D. Hwang and G. K. Yu, "Shear-wave velocity structure of upper mantle under Taiwan from the array analysis of surface waves," Geophysical Research Letters, vol. 32, no. 7, 2005.

[6] F. Garofalo, S. Foti, F. Hollender et al., "InterPACIFIC project: comparison of invasive and non-invasive methods for seismic site characterization. part II: inter-comparison between surface-wave and borehole methods," Soil Dynamics and Earthquake Engineering, vol. 82, no. 1, pp. 241-254, 2016.

[7] K. H. Stokoe, S. H. Joh, and R. D. Woods, "Some contributions of in situ geophysical measurements to solving geotechnical engineering problems," in Proceedings of the ISC2: 2nd International Conference on Geotechnical and Geophysical Site Characterization, pp. 19-22, Viana da Fonseca, Porto, Portugal, September 2004.

[8] S. Foti, S. Parolai, D. Albarello, and M. Picozzi, "Application of surface-wave methods for seismic site characterization," Surveys in Geophysics, vol. 32, no. 6, pp. 777-825, 2011.

[9] V. Di Fiore, G. Cavuoto, D. Tarallo, M. Punzo, and L. Evangelista, "Multichannel analysis of surface waves and down-hole tests in the archeological "Palatine hill" area (Rome, Italy): evaluation and influence of $2 \mathrm{D}$ effects on the shear wave velocity," Surveys in Geophysics, vol. 37, no. 3, pp. 625-642, 2016.

[10] T.-F. Zhou, G.-X. Peng, T.-Y. Hu, W.-S. Duan, F.-C. Yao, and Y.-M. Liu, "Rayleigh wave nonlinear inversion based on the Firefly algorithm," Applied Geophysics, vol. 11, no. 2, pp. $167-178,2014$.

[11] ASTM D7400-4 2007, Standard Test Methods for Downhole Seismic Testing: Annual Book of ASTM Standard, ASTM, West Conshohocken, PA, USA, 2007.

[12] Z. Y. Lin, Geotechnical Testing and Monitoring Manual, Liaoning Science and Technology Publishing, Shenyang, China, 1994.

[13] E. J. Baziw, "Digital filtering techniques for interpreting seismic cone data," Journal of Geotechnical Engineering, vol. 119, no. 6, pp. 998-1018, 1993.

[14] X. M. Hou, Y. X. Shan, L. Z. Peng, and B. J. Shen, “An optimized approach for single-hole method of shear wave velocity measurement based on correlation functions," Rock and Soil Mechanics, vol. 27, pp. 1161-1165, 2006, in Chinese.

[15] D. Crice, Borehole Shear-Wave Surveys For Engineering Site Investigations, Geostuff, Saratoga, NY, USA, 2002.

[16] R. G. Campanella and W. P. Stewart, "Seismic cone analysis using digital signal processing for dynamic site characterization," Canadian Geotechnical Journal, vol. 29, no. 3, pp. 477-486, 1992.

[17] K. Ishihara, Soil Behaviour in Earthquake Geotechnics, Oxford University Press, Oxford, UK, 1996.

[18] Z. P. Liao, Introduction to Wave Motion Theories in Engineering, Science Press, Beijing, China, 1996.

[19] Z. P. Liao and H. L. Wong, "A transmitting boundary for the numerical simulation of elastic wave propagation," International Journal of Soil Dynamics and Earthquake Engineering, vol. 3, no. 4, pp. 174-183, 1984. 
[20] Z.-P. Liao, "Extrapolation non-reflecting boundary conditions," Wave Motion, vol. 24, no. 2, pp. 117-138, 1996.

[21] I. Sanchez-Salinero, J. M. Roesset, and K. H. Stokoe, Analytical Studies of Body Wave Propagation and Attenuation, Texas University at Austin Geotechnical Engineering Center, Austin, TX, USA, 1986.

[22] K. Aki and P. G. Richards, Quantitative Seismology, University Science Book, Herndon, VA, USA, 1980.

[23] C.-C. Chao, "Dynamical response of an elastic half-space to tangential surface loadings," Journal of Applied Mechanics, vol. 27, no. 3, pp. 559-567, 1960.

[24] S. Teachavorasinskun and P. Lukkunaprasit, "A simple correlation for shear wave velocity of soft Bangkok clays," Géotechnique, vol. 54, no. 5, pp. 323-326, 2004.

[25] X. M. Hou, J. S. Bo, X. S. Yang, and D. Liu, "Applications of correlation function in shear-velocity measurement of singlehole method," Earthquake Engineering and Engineering Dynamics, vol. 24, pp. 60-65, 2004, in Chinese.

[26] L. Jin and J. Liang, "Dynamic soil-structure interaction with a flexible foundation embedded in a half-space: closed-form analytical solution for incident plane SH waves," Journal of Earthquake Engineering, vol. 25, no. 8, pp. 1565-1589, 2021. 